Cahiers $d u$ MONDE RUSSE

\section{Cahiers du monde russe}

Russie - Empire russe - Union soviétique et États indépendants

$48 / 4 \mid 2007$

Varia

\title{
Gaston Paris et Aleksandr Veselovskij
}

Les relations scientifiques franco-russes, une page d'histoire

\section{Michel ZINK et Petr ZABOROV}

\section{(2) OpenEdition}

1 Journals

Édition électronique

URL : https://journals.openedition.org/monderusse/9034

DOI : $10.4000 /$ monderusse. 9034

ISSN : $1777-5388$

Éditeur

Éditions de l'EHESS

\section{Édition imprimée}

Date de publication : 2 décembre 2007

Pagination : 637-665

ISBN : 978-2-7132-2148-4

ISSN : $1252-6576$

Référence électronique

Michel ZINK et Petr ZABOROV, "Gaston Paris et Aleksandr Veselovskij 》, Cahiers du monde russe [En ligne], 48/4 | 2007, mis en ligne le 01 janvier 2007, consulté le 02 septembre 2022. URL : http:// journals.openedition.org/monderusse/9034; DOI : https://doi.org/10.4000/monderusse.9034 
chercher : repérer : avancer

Cet article est disponible en ligne à l'adresse :

http://www.cairn.info/article.php?ID REVUE=CMR\&ID NUMPUBLIE=CMR 048\&ID ARTICLE=CMR $048 \quad 0637$

\section{Gast on Paris et Aleksandr Veselovskij. Les relations scientifiques franco- russes, une page d'histoire}

par Michel ZINK et Petr ZABOROV

\section{Editions de l'EHESS | Cahiers du monde russe}

$2007 / 4-\mathrm{n}^{\circ} 48$

ISSN 1252-6576 | ISBN 9782713221484 | pages 637 à 665

Pour citer cet article :

-ZINK M. et ZABOROV P., Gast on Paris et Aleksandr Veselovskij. Les relations scientifiques franco-russes, une page d'hist oire, Cahiers du monde russe 2007/ 4, n 48, p. 637-665.

Distribution électronique Cairn pour les Editions de l'EHESS.

(C) Editions de l'EHESS. Tous droits réservés pour tous pays.

La reproduction ou représentation de cet article, notamment par photocopie, n'est autorisée que dans les limites des conditions générales d'utilisation du site ou, le cas échéant, des conditions générales de la licence souscrite par votre établissement. Toute autre reproduction ou représentation, en tout ou partie, sous quelque forme et de quelque manière que ce soit, est interdite sauf accord préalable et écrit de l'éditeur, en dehors des cas prévus par la législation en vigueur en France. Il est précisé que son stockage dans une base de données est également interdit. 


\title{
GASTON PARIS ET ALEKSANDR VESELOVSKIJ
}

\author{
Les relations scientifiques franco-russes, \\ une page d'histoire
}

\section{Avant-propos de Michel Zink Introduction et annotations de Petr Zaborov}

Gaston Paris et Aleksandr Veselovskij auraient pu se rencontrer en Russie, où en 1856, à l'issue de ses études secondaires, Gaston Paris accompagne ses parents. Il s'agit de rendre visite à ses sœurs aînées, qui y sont pour un temps toutes deux installées avec leurs maris négociants. Son père Paulin Paris, professeur au Collège de France depuis 1852, est en outre chargé par le ministère de l'Instruction publique d'une mission de recherche et de consultation des manuscrits français dans les bibliothèques de Saint-Pétersbourg et de Moscou. Gaston Paris profite de ce séjour de trois mois pour étudier le russe ${ }^{1}$. Vingt-et-un ans plus tard, il ne laissera pas ignorer à Aleksandr Veselovskij qu'il a quelque connaissance de cette langue ${ }^{2}$.

Mais à quoi bon récrire l'histoire ? La Russie est vaste. Les deux jeunes gens (Gaston Paris avait alors dix-sept ans, Veselovskij dix-huit) ne s'y sont pas croisés. Ils n'entreront en correspondance que douze ans plus tard. Lorsque Veselovskij lui écrit pour la première fois, en janvier 1868, la position proprement universitaire de Gaston Paris est encore modeste. C'est seulement l'année suivante, dès la création de cette institution, qu'il commencera son enseignement à l'École pratique des hautes études. Mais son grand livre, Histoire poétique de Charlemagne, a paru dès 1865. Il est déjà une figure connue, comme le montre le fait qu'à partir de cette même année 1868 il est convié aux fameux dîners Magny³ ${ }^{3}$ Durant l'année universitaire 1866-1867, il a pour la première fois remplacé son père dans son enseignement

1. Ursula Bähler, Gaston Paris et la philologie romane, Genève : Droz, 2004, p. 37-38.

2. Voir ci-dessous lettre 3.

3. Bähler, op. cit., p. 127. 
au Collège de France. Enfin, il a fondé en 1866 avec Paul Meyer, Charles Morel et Hermann Zotenberg la Revue critique d'histoire et de littérature : c'est à l'occasion de l'envoi de son premier livre à la revue que Veselovskij lui adresse sa première lettre. Elle ne semble pas avoir marqué le début de relations suivies ni d'une véritable correspondance, puisque les premiers mots d'une lettre de Veselovskij huit ans plus tard, en avril 1874, sont : «Monsieur, Si je prends la liberté de m'adresser à vous sans avoir eu l'honneur de vous connaître personnellement [...]». Entre temps, Gaston Paris est devenu le maître de la philologie romane. En 1872, il a succédé à son père comme professeur au Collège de France et il a fondé avec Paul Meyer la revue Romania, où il se réjouit en 1877 de pouvoir publier le Dit de l'empereur Constant édité par Aleksandr Veselovskij4.

À partir de ce moment, la correspondance entre les deux savants, qui se poursuit à intervalles espacés jusqu'en 1899 (Gaston Paris mourra en 1903, Veselovskij en 1906), témoigne d'une chaleur, d'une estime et d'une confiance grandissantes entre eux. Leurs lettres n'auraient cependant qu'un intérêt anecdotique sans l'importance de leur personnalité à l'un et à l'autre (encore Gaston Paris ne semble-t-il pas mesurer pleinement la puissance intellectuelle de son correspondant) et surtout si l'on ignorait le rôle et la position d'Aleksandr Veselovskij dans l'un des plus grands débats scientifiques de la philologie romane à cette époque, celui dans lequel Gaston Paris s'est le plus fortement impliqué : la question de l'origine des chansons de geste.

Dans son Histoire poétique de Charlemagne, Gaston Paris supposait que les événements qui, à l'époque carolingienne, avaient frappé les imaginations (par exemple le désastre de Roncevaux) avaient immédiatement donné naissance à des poèmes brefs, des cantilènes, longtemps diffusées oralement avant d'être rassemblées et refondues pour donner naissance aux longs poèmes que sont les chansons de geste. Il applique ainsi aux épopées romanes les théories alors en vogue touchant la composition des poèmes homériques. En 1884, le philologue italien Pio Rajna publie ses Origini dell'epopea francese. Sans être en désaccord sur tous les points avec Gaston Paris, il met en doute l'existence de cantilènes romanes telles que les imaginait Gaston Paris et insiste surtout sur les origines germaniques des chansons de geste. Gaston Paris se rallie pour l'essentiel à ses vues dans un très long compte rendu publié la même année dans Romania.

L'ouvrage, pourtant capital, de Pio Rajna suscita peu d'autres réactions du côté français, à l'exception d'un compte rendu très élogieux d'Arsène Darmesteter dans la Revue critique de 1884. Mais l'année suivante Aleksandr Veselovskij en publia un à son tour, très long, très critique, mais extrêmement suggestif, dans la Revue $d u$ ministère de l'Instruction publique d'avril 1885 (p. 253-285) ${ }^{5}$. Il formidabile

4. Romania,t. 6, 1877, p. 161-198.

5. Je dois toute mon information sur ce compte rendu et sur la position de Veselovskij à l'article de Patrizia Gasparini, «Le rôle de la tradition dans la circulation de la littérature médiévale : Gaston Paris, Pio Rajna et Aleksandr N. Veselovskij », in Michel Zink, éd., Le Moyen Âge de Gaston Paris. La poésie à l'épreuve de la philologie, P. : Odile Jacob, 2004, p. 131-160. 
Russo, comme l'appelait Carlo Dionisotti 6 , y prenait ses distances aussi bien avec Gaston Paris qu'avec Pio Rajna, en réservant cependant à ce dernier l'essentiel de ses traits. Le détail du débat importe peu ici. Mais le socle de l'argumentation de Veselovskij mérite d'être rappelé. Il dénonce une approche trop historique de l'origine des chansons de geste et une confusion entre des poèmes épiques déjà constitués d'une part, et de l'autre l'universalité de l'imagination, de la poétique et des légendes épiques. Les motifs sur lesquels Pio Rajna croyait pouvoir s'appuyer pour reconstituer une filiation des poèmes épiques (combat du père contre le fils, discours hautain des ambassadeurs, utilisation d'un philtre soporifique pour s'emparer de quelque chose, etc.), il montre qu'on ne les trouve pas seulement dans les épopées germaniques et dans les chansons de geste romanes, mais partout, et qu'ils demandent à être interprétés dans une perspective, non seulement comparatiste, mais aussi, comme nous dirions aujourd'hui, anthropologique. Il réclame une typologie des motifs. Il juge l'épopée médiévale historique, non en ce qu'elle mentionne des faits historiques, mais en ce qu'elle reflète les mouvements de l'âme populaire à travers les siècles de l'histoire.

S'il élargit ainsi le débat et si la minutieuse enquête positiviste de Rajna lui paraît étriquée, c'est probablement parce qu'il est Russe et qu'il a, s'agissant des traditions et de la poésie populaires, un horizon culturel beaucoup plus vaste que ses collègues français ou italiens. On voit aussi que, dès ce moment-là et sur un sujet que tous les autres savants de son temps considèrent comme relevant uniquement de la philologie et de l'histoire littéraire, il est le précurseur de Propp. Mais il prêche dans le désert.

Sa correspondance avec Gaston Paris, aujourd'hui reconstituée grâce à l'heureuse découverte de ses lettres complétant celles, déjà connues, du savant français, ne dépasse guère le niveau des bonnes manières entre collègues. C'est peutêtre que, sur les questions les plus fondamentales posées par leurs études, Veselovskij était seul.

Michel Zink, Collège de France, chaire de littératures de la France médiévale Institut de France, Académie des inscriptions et belles-lettres

6. Gasparini, art. cit., p. 242. 


\section{Introduction}

Gaston Paris (1839-1903), un des principaux médiévistes français, professeur au Collège de France et à l'École pratique des hautes études, membre de l'Académie des inscriptions et belles-lettres de l'Académie française, ainsi que de nombreuses sociétés savantes, enfin fondateur avec Paul Meyer de la revue Romania, jouissait d'une grande notoriété aussi bien en France que dans le monde. Dans son article intitulé «In memoriam. Gaston Paris », Melchior de Vogüé soulignait en ces termes son autorité internationale : «À Edimbourg comme à Stockholm, à Berlin comme à Moscou, le nom de Paris était grand entre les plus grands de France ${ }^{1}$.

Saint-Pétersbourg aurait pu figurer parmi ces villes choisies un peu au hasard : Aleksandr Veselovskij (1838-1906), professeur à l'université de Saint-Pétersbourg et académicien, y travailla de 1870 à la fin de sa vie ${ }^{2}$. Son érudition exceptionnelle, sa connaissance d'un grand nombre de langues, l'étendue de ses intérêts scientifiques (linguistique, ethnographie, poétique historique, étude comparée des cultures, etc.), en firent un phénomène proprement remarquable dans la vie intellectuelle russe. «Il ressemblait, d'une certaine façon, à un homme de la Renaissance, qui brillait d'une sorte de jeunesse d'esprit éternelle et lumineuse », écrivait son cadet V.N. Peretc, professeur à l'université de Kiev et futur académicien, qui ne craignit pas d'utiliser à son propos la formule : «Un Mozart de la science $»^{3}$. Veselovskij joua un grand rôle dans la vie universitaire russe, car c'est sous sa direction et sous son influence que furent formés presque tous les spécialistes de langues et littératures romanes et germaniques à la fin du XIX ${ }^{\mathrm{e}}$ et au début du XX $\mathrm{X}^{\mathrm{e}}$ siècle.

Le domaine de prédilection de Veselovskij étaient le Moyen Âge et la Renaissance qui faisaient l'objet de la plupart de ses travaux : sa thèse de maîtrise et en partie sa thèse de doctorat, ses ouvrages sur Boccace et Pétrarque, ses cours, ses cycles d'articles et collections ; il était aussi l'auteur d'une excellente traduction du Décaméron. Cette orientation générale le conduisit à des relations avec des collègues étrangers, dont il suivait attentivement les travaux, s'efforçant de ne manquer aucune publication tant soit peu importante, que ce fût un ouvrage en plusieurs volumes ou une note dans une revue ${ }^{4}$.

Veselovskij correspondit avec beaucoup de ses collègues, dont il connaissait certains personnellement ; assez souvent, ces contacts professionnels débouchèrent sur

1. Eugène Melchior de Vogüé, Sous l'horizon, hommes et choses d'hier, P. : A. Colin, 1904, p. $253-254$

2. Voir I.K. Gorskij, Aleksandr Veselovskij i sovremennost' [Alksandr Veselovskij et l'époque actuelle], M.: Nauka, 1975 ; P.R. Zaborov, éd., Nasledie Aleksandra Veselovskogo. Issledovanija i materialy, [L'héritage d'Aleksandr Veselovskij. Recherches et matériaux], SPb.: Nauka, 1992. Voir, également, Charles Ridoux, Évolution des études médiévales en France de 1860 à 1914, P.: Champion, 2001, p. 603-604.

3. Čtenija v Istoričeskom ob̌̌čestve Nestora-letopisca [Lectures à la société d'histoire du chroniqueur Nestor], Kiev, 1908, vol. XX, n¹, section 1, p. 29.

4. Voir M.P. Alekseev, « A.N. Veselovskij i zapadnoe literaturovedenie » [A.N. Veselovskij et les études littéraires occidentales], Izvestija Akademii Nauk SSSR, Otdelenie obščestvennyh nauk, $n^{\circ} 4$, p. 121-138. 
une sympathie mutuelle, voire une amitié. Il eut des relations particulièrement étroites et prolongées avec Alessandro D'Ancona, spécialiste littéraire, critique et journaliste italien, avec le slaviste d'origine croate Vatroslav Jagič, professeur aux universités de Berlin et de Vienne, qui avait longuement vécu en Russie, et enfin avec Gaston Paris.

Veselovskij et Paris se rencontraient rarement et ne s'écrivaient pas très souvent, mais leur relation dura pendant plus de trente ans, elle fut parfois très nourrie et l'intérêt de Paris pour Veselovskij ne fit que croître, ce qui transparaît dans ses réactions aux travaux de son collègue (ce qui n'était guère facile pour Paris, étant donné sa faible connaissance de la langue russe). C'est ainsi qu'en 1888 il écrivait que « le Moyen Âge français est devenu, pour la plupart des nations civilisées, comme une seconde antiquité », et relevait parmi elles la Russie « où M. Wesselofsky, le profond connaisseur des littératures du Moyen Âge est en train de fonder une école de philologie romane $»^{5}$. Répondant à la prière de Veselovskij, Paris donnait aussi volontiers des conseils et des renseignements aux élèves de celui-ci, qui évoquaient ensuite en des termes enthousiastes, dans leurs lettres à leur maître, leurs entretiens avec Paris, ses conférences au Collège de France et ses séminaires à l'École pratique des hautes études ${ }^{6}$.

On sait peu de choses sur les rencontres entre les deux savants, mais leur correspondance s'est conservée sinon in extenso, du moins sans graves lacunes. Ce sont 12 lettres de Paris, y compris deux cartes de visite, qui se trouvent à la Maison Pouchkine de l'Académie des sciences de Russie à Saint-Pétersbourg (fonds $n^{\circ} 45$ ) et 8 lettres de Veselovskij récemment trouvées à la Bibliothèque nationale de France (MSS, NAFr. 24463. Correspondance Gaston Paris, vol. XXXIV). Les lettres de Paris ont été pour la plupart publiées ${ }^{7}$, ce qui n'est pas le cas de celles de Veselovskij. C'est seulement aujourd'hui qu'il devient possible de retracer l'histoire de cette amitié professionnelle.

Toutes les lettres sont publiées d'après les autographes; nous avons corrigé les erreurs qui se sont glissées dans les textes de Veselovskij et qui viennent pour l'essentiel de l'influence de l'orthographe italienne. Les mots donnés sous forme abrégée sont rétablis dans leur intégralité avec des crochets brisés, de même que les caractères ou les dates qui sont omises dans les originaux, marquées ici entre des crochets droits. L'auteur remercie André Berelowitch pour ses précieux conseils.

Institut russkoj literatury
Puškinskij Dom
zaborov@pz7588.spb.edu

5. Journal des savants, novembre 1888, p. 664.

6. Voir, par exemple, la lettre de Fedor Dmitrievič Batjuškov (évoqué ci-dessous) à Veselovskij, Paris, le 18 mars 1888 et la remarque de Veselovskij dans sa réponse du 4 avril (ancien style) : «Je savais d'avance que G. Paris vous plairait ; il les dépasse tous et de sa tête, et de son talent, et par la variété de ses intérêts. » Maison Pouchkine, ms., n 15 041,f. 107.

7. Certaines des lettres présentées ci-après $(6,8,9,10,12,14)$ ont déjà été publiées «Iz pisem Gastona Parisa k akademiku A.N. Veselovskomu » [Lettres de Gaston Paris à l'académicien Veselovskij], par P.R. Zaborov, Izvestija Akademii Nauk SSSR, Serija literatury i jazyka, vol. 40, 5, p. 456-464 ; P.R. Zaborov, «A.N. Veselovskij i francuzskie učenye (po arhivnym materialam) » [A.N. Veselovskij et les savants français (d'après des archives)], Russkaja literatura, ${ }^{\circ} 1,1988$, p. 140-142. 
Alexandre Wesselofsky à Gaston Paris

3 (15) janvier 1868.

Monsieur,

Permettez-moi de vous offrir le $1^{\mathrm{r}}$ volume du texte italien qui vient de paraître à Bologne et que je vois annoncé au premier $N^{\circ}$ de votre Revue critique de $1868^{1}$. Il n'était pas dans mon intention de le donner au public tel que vous le voyez, détaché qu'il est du $2^{\mathrm{d}}$ volume, qui doit contenir la partie la plus intéressante du texte, et de la préface et des documents, qui doivent en relever la portée historique. Mais monsieur Romagnoli l'a voulu, et il est difficile de conter avec les éditeurs ${ }^{2}$. Ce qui me donne le plus de peine est l'appréhension que la lecture du $1^{\mathrm{r}}$ volume, qui est le plus ennuyeux, ne décourage le lecteur et ne lui ôte le désir de continuer. Car le style de mon auteur n'est pas de ceux qu'on goûte généralement en Italie : ce n'est pas certes la langue del buon secolo ${ }^{3}$, mais une langue de transition, l'auteur se souvenant dans sa vieillesse et dans le premier quart du Xve siècle, au milieu de la littérature latine naissante de la période des Médicis des traditions littéraires du siècle passé et des entretiens à la villa del Paradiso, où, jeune encore, il a pu entendre Luigi de' Marsili et Coluccio Salutati, les amis et les disciples de Pétrarque et de Boccace ${ }^{4}$. Ainsi placé sur le confin de deux siècles, il se ressent de l'un et de l'autre, dans la langue comme dans les idées.

Une autre cause ajoute beaucoup à l'imperfection du style : c'est l'état du manuscrit, dont notre texte n'est qu'une reproduction fidèle. C'est l'autographe même de l'auteur, un brouillon, tout bigarré de corrections interlinéaires, de renvois et de notes à la marge, qui en font difficile la lecture. L'auteur essaie la même phrase à plusieurs reprises, et parfois il oublie de rayer à côté de la rédaction qu'il accepte définitivement, quelque mot de la rédaction précédente qu'il a rejetée. D'autres fois, tout en faisant le même travail de révision, il raie par mégarde un mot

1. Revue critique d'histoire et de littérature. Elle fut fondée en 1866 à Paris par G. Paris, P. Meyer, Ch. Morel et H. Zotenberg ; l'annonce dont il est question était imprimée au verso de la page de titre du premier numéro de l'année 1868 (Bulletin bibliographique des principales éditions françaises et étrangères).

2. Il s'agit de la version en italien de la thèse de maîtrise d'Aleksandr Veselovskij qui était la publication scientifique d'une œuvre italienne du XIVe siècle qu'il avait découverte à la Biblioteca Riccardiana de Florence : Il Paradiso degli Alberti, ritrovi e ragionamenti del 1389, romanzo di Giovanni da Prato dal codice autografo e anonimo della Riccardiana a cura di Alessandro Wesselofsky, Bologne : Presso Gaetano Romagnoli, 1867. Le premier tome de cette œuvre en trois volumes contenait l'appareil critique, le second et le troisième tomes contenaient le texte. Cependant, et en dépit du vœu de Veselovskij, Gaetano Romagnoli, professeur à l'université de Bologne qui publiait cette œuvre, fit paraître d'abord le second tome, c'est-àdire la majeure partie du texte, et seulement ensuite le premier et le troisième tomes, à savoir l'appareil critique et la fin du texte. D'où l'explication au début de cette lettre.

3. Buon secolo - le XIve siècle.

4. Luigi de Marsili (1330-1394), diplomate et théologien florentin ; Coluccio Salutati (13311406), humaniste et poète florentin. 
de l'ancienne rédaction, qui devait faire partie de la rédaction définitive. C'est ainsi qu'il m'est échappé d'écrire avec celle-ci, page 29, 15 « chiare fontane, con mille rivoli pesci notate », où $\mathrm{Mr}$. Carducci ${ }^{5}$ me proposait de lire notante, pour remédier un peu à la grammaire. Or, la première rédaction de l'auteur portait : « con mille rivoli da innumerabili pesci notate»; une seconde: «da infiniti p<esci> $\mathrm{n}<$ otate $>$ » en rayant l'une et l'autre, il en a fait autant de la préposition da, qu'il faut rétablir dans le texte, comme je le fais remarquer dans les errata et le glossaire joint au $2^{\mathrm{d}}$ volume $^{6}(\mathrm{a}<\mathrm{d}>\mathrm{v}<$ ocem $>$ notato, usé dans le sens actif, comme Ovid. Pist. 179 : « ̇̀ manifesta la mia usata via delle notate acque $»)^{7}$.

Le Ms. est sans titre et sans nom d'auteur : il m'a fallu lui donner l'un et tâcher de retrouver l'autre. Je ne sais si j'ai réussi à cette dernière recherche ; mais je crois d'avoir pu fixer avec assez de précision l'époque à laquelle doivent se rapporter ces réunions littéraires du Paradis, que l'auteur nous a décrites : les biographies de 15 personnages historiques contemporains, qui viennent y figurer successivement, m'ont facilité la tâche.

Il restait encore une chose à faire : il s'agissait de dévoiler un plagiaire, qui à la fin du siècle passé s'est servi de mon Ms. pour en extraire quelques nouvelles détachées, et les publier, en en rajeunissant le style, sous le nom d'un auteur imaginaire de la fin du $\mathrm{XV}^{\mathrm{e}}$ siècle $^{8}$. Heureusement ce monsieur a eu l'étrange idée de passer pour un honnête homme, et pour faire preuve de sa véracité et de la fidélité avec laquelle il aurait reproduit l'original, il a imaginé des lacunes dans son texte et reproduit par mégarde d'autres, qui se retrouvent ligne par ligne dans mon manuscrit. Aussi ai-je consacré les deux premiers chapitres de mon travail à dévoiler la fraude, en faisant en même temps l'histoire du plagiat et du manuscrit qui lui a servi de base.

J'ai cru, Monsieur, en vous envoyant mon livre, devoir vous soumettre ces quelques observations, puisque après tout il s'agissait pour moi que les premières pages ne vous décourageassent de le lire jusqu'au bout : c'est à quoi je tiens beaucoup.

Si vous aviez quelques recherches à faire dans les bibliothèques de Heidelberg, de Stuttgart ou de Carlsruhe, où je me trouve présentement ${ }^{9}$, veuillez vous servir de votre très dévoué

Alexandre Wesselofsky. 1868, le 15 janvier. Gr<and> duché de Bade, Carlsruhe, Lange Strasse, 74. BNF, MSS, NAFr. 24463, f. 118-119. Cette référence sera donnée infra sous forme abrégée.

5. Giosuè Carducci (1835-1907), poète et prix Nobel italien, spécialiste littéraire, depuis 1866 professeur à l'université de Bologne.

6. Il Paradiso degli Alberti [...], vol. 2, p. 29. Voir note vol. 1, partie 2.

7. Nous ignorons la source de cette citation.

8. Il s'agit de Favola composta per Giraldo Giraldi nel tempo della moria nell'anno 1479, Amsterdam (en fait Florence), 1796, publié par Gaetano Cioni.

9. À Karlsruhe, Veselovskij était précepteur de Serge, fils du duc Maximilien de Leuchtenberg et de la grande duchesse Marija Nikolaevna, la fille de Nicolas Ier. 
Alexandre Wesselofsky à Gaston Paris

8 (20) avril 1874.

Monsieur,

Si je prends la liberté de m'adresser à vous sans avoir eu l'honneur de vous connaître personnellement, c'est que je compte sur votre complaisance et que ma requête ne sera pas longue et ne vous prendra pas beaucoup de temps.

Parmi les employés de la bibliothèque Nationale il ne vous sera pas difficile de trouver quelqu'un, qui veuille bien me copier une demi-page d'ancien français. Il s'agit des jeûnes $d u$ Vendredi, dont le commencement a été donné par $\mathrm{M}$. $\mathrm{P}<$ aulin> Paris : Mss. franç . IV, p. 18 (ms. $7019^{3}$ f. 3) ${ }^{10}$; j'en voudrais avoir la suite pour m'en servir dans un petit travail qui doit paraître prochainement ${ }^{11}$. Quant aux frais de la transcription, j'en enverrai le montant aussitôt que j'en suis avisé.

Si jamais je suis en état de vous être utile dans un cas pareil, je le ferai avec le plus grand empressement.

Agréez, Monsieur le professeur, l'assurance de mon profond dévouement.

Alexandre Wesselofsky. Russie, St. Pétersbourg, Université.

BNF,f. 120-121.

Gaston Paris à Alexandre Wesselofsky

[Avril 1877.]

Cher Monsieur,

Votre envoi est arrivé fort à point. Je m'évertuais à faire un article français pour le prochain $\mathrm{n}^{\circ}$ de la Romania, et je ne trouvais pas le temps nécessaire, quand l'empereur Coustant est venu me tirer d'embarras ${ }^{12}$. Aussi est-il parti tout de suite pour l'imprimerie. Vous possédez si bien notre langue que j'ai eu bien peu de

10. Voir A. Paulin Paris, Les manuscrits français de la Bibliothèque du Roi, 1841, t. IV, p. 18.

11. Il s'agit de l'article « Skazanie o 12-ti pjatnicah » [La légende des 12 vendredis ], qui entrait dans le cycle Opyty po istorii razvitija hristianskoj legendy [Essais sur l'évolution des légendes chrétiennes], Zurnal ministerstva narodnogo prosveščenija, vol. 185, juin 1876, p. 326-367. Voir les remerciements de Veselovskij à Gaston Paris pour lui avoir procuré ce texte complet Ibid., p. 333.

12. Article de Veselovskij «Le dit de l'empereur Constant», Romania, t. VI, n² 22, 1877, p. 161-198. 
corrections à faire. Vous m'excuserez, je pense, comme j'ai revu avec soin le texte, d'avoir mentionné ce petit fait sous votre signature ${ }^{13}$.

J'ai été surpris, avant-hier, de voir entrer l'excellent Sundby ${ }^{14}$, qui traversait Paris allant en Espagne. Il m'a dit qu'il avait vu avec surprise sur la couverture de la Romania l'annonce de votre publication, qu'il vous avait en effet autorisé à imprimer sa copie, mais qu'il pensait que vous l'auriez fait dans un livre et non dans un journal, que cela, il aurait bien pu le faire lui-même ; qu'il n'était pas un copiste, etc. Je lui ai dit ce que j'ai pu ; mais vous ferez peut-être bien de m'envoyer deux ou trois lignes plus explicites pour le remercier de son obligeance, dire qu'il a fait non une simple copie, mais un texte préparé pour l'impression, etc., puisqu'il y tient ${ }^{15}$. C'est un homme d'un très grand mérite et très aimable, un peu susceptible peut-être.

J'espère que vous ne vous en tiendrez pas là dans votre collaboration à la Romania. Cela pourrait vous servir à acheter des livres à Paris.

Est-il vrai que Kirpitchnikof soit à Berlin ?16 Si ce n'est pas vrai, et que vous le voyez, faites lui, je vous prie, toutes mes amitiés.

Je n'ai pas fait en russe, pendant son séjour, les progrès que j'espérais, cependant je lis un peu plus facilement. Ne craignez donc pas de m'envoyer ce que vous publierez en russe.

Ne désirez-vous avoir un tirage à part de votre mémoire, si intéressant ? Nos conditions sont : 50 exemplaires, payés par l'auteur (le papier) et non mis dans le commerce. Peut-être vous contenteriez-vous de quelques exemplaires des feuilles qui contiendront l'article. Veuillez me le faire savoir.

Croyez, cher Monsieur, à mes sentiments bien distingués et tout dévoués.

G. Paris

7, rue du Regard.

Maison Pouchkine (MP), f. 45, op. 3, N 593, 1. 14-15. Cette réference sera donnée infra sous forme abrégée.

\section{4}

Alexandre Wesselofsky à Gaston Paris

\section{Monsieur,}

Je vous suis infiniment obligé de l'accueil favorable que vous avez fait à mon petit essai. Quant au texte, que $\mathrm{M}^{\mathrm{r}}$ Sun[d]by a eu l'obligeance de me communiquer, en voici l'histoire. Ne connaissant $M^{r} \operatorname{Sun}[\mathrm{d}]$ by que par ses écrits, je me suis

13. Ce fait est mentionné par Veselovskij dans l'introduction de son article (note 17).

14. Thor Sundby (1830-1894), professeur de langue et de littérature françaises à l'université de Copenhague.

15. Veselovskij exauça cette demande de G. Paris en préambule à son article (voir note 18).

16. Aleksandr Ivanovič Kirpičnikov (1845-1903), professeur de lettres à l'université de Kharkov, puis d'Odessa, puis de Moscou. L'été 1874, au cours de sa période probatoire, il était stagiaire chez G. Paris qui, en retour, en profita pour perfectionner sa connaissance de la langue russe. 
adressé à $\mathrm{M}^{\mathrm{r}}$ le prof. Smith à Copenhague ${ }^{17}$, en le priant de me faire copier, par quelque personne compétente, le Dit en question. $\mathrm{M}^{\mathrm{r}} \mathrm{Smith}$ en parla à $\mathrm{M}^{\mathrm{r}} \mathrm{Sun}[\mathrm{d}]$ by, qui a eu la bonté d'exécuter lui-même la copie requise. Il l'a fait suivre d'une lettre, dont voici le commencement : «N'ayant pas encore reçu la Revue que vous avez bien voulu m'offrir (il s'agit de mon article sur les “Constantinische Sagen” dans la Russische Revue), je ne connais pas votre notice sur l'histoire traditionnelle de Constantin; mais cela n'empêche pas que je ne vous regarde comme l'éditeur naturel du Dit de Coustant, dont je vous ai envoyé une copie il y a trois mois. Quant à moi, je ne pense pas à publier ce petit texte, qui me paraît du reste assez curieux. Je l'ai copié pour vous, Monsieur, et je vous prie d'en disposer à votre gré ».

Il est heureux pour moi que la lettre de $\mathrm{Mr}^{\mathrm{r}} \operatorname{Sun}[\mathrm{d}]$ by se soit conservée ; je la garde encore ${ }^{18}$.

Je vous remercie de l'avis que vous me donnez à cet endroit et j'écrirai aujourd'hui même à $\mathrm{M}^{\mathrm{r}} \mathrm{Sun}[\mathrm{d}] \mathrm{by}$. Je crois qu'il serait bien de faire ressortir avec éclat, dans quelques lignes qui serviraient d'introduction au texte, la part de Mr Sun[d]by - la mienne étant des plus minimes. Je ne voudrais pour rien au monde que mon apparition dans la Romania soulevât des susceptibilités ; n'ayant nulle envie d'en grossir la liste, je laisserai plutôt tomber mon article ${ }^{19}$.

Comme je n'ai pas de tirage à part de mon écrit, inséré dans la Russ<ische> Revue, j'en voudrais avoir de la Romania (50 exemplaires). Le premier et le meilleur sera pour Mr Thor Sun[d]by.

Mr Kirpitschnikoff est présentement à Saint-Pétersbourg. J'irai le voir un de ces jours ; pour le moment je suis hors d'état de voir ou de fréquenter qui que ce soit, car je figure depuis une semaine, et pour 15 jours encore, en qualité de juré.

Agréez, cher Monsieur, l'expression de mes sentiments les plus distingués.

Alexandre Wesselofsky.

PS. Je ne sais si vous trouverez convenable de me faire dire quelque part au bas de la page, que mon article a été refait sur une première rédaction allemande. Voici de quoi il s'agit: dans mon écrit allemand j'ai renvoyé au travail, que vous connaissez, de $\mathrm{Mr}$ A. Weber, que je considère comme mon devancier, lui cédant volontiers le pas et l'honneur ${ }^{20}$. Dans la rédaction française cette note a été omise,

17. Sophus Birket-Smith (1838-1919), spécialiste de lettres et historien.

18. Cette lettre (Copenhague, 2 octobre 1875) se trouve dans les archives de Veselovskij (MP, f. 45, inv. 3, $\mathrm{N}^{\circ} 733$ ). L'article de Veselovskij dont il s'agit fut publié dans la Russische Revue (1875, IV Band, p. 178-207).

19. «Par la gracieuse entremise de M. le prof. Smith, écrivait Veselovskij, M. Thor Sundby eut la bonté d'en prendre pour moi une copie : j'offre ici tous mes remerciements à l'éminent savant qui m'a permis d'être à sa place l'éditeur de ce poème ; il est inutile de le dire que sa copie était faite, non seulement avec exactitude, mais avec l'intelligence que peut seule donner une connaissance approfondie de l'ancien français. Je préparai cette copie pour l'impression, et M. G. Paris a pris la peine de la revoir et d'en corriger les épreuves » (Romania, vol. VI, $n^{\circ} 22,1877$, p. 162).

20. Alfred Weber (1835-1914), philologue suisse, spécialiste de langues et littératures romanes. Il est notamment l'auteur de Handschrifliche Studien auf den Gebiete romanischen Literatur des Mittelalters, Frauenfeld, Suisse, 1876. 
comme étant désormais inutile. Mais la susceptibilité de $\mathrm{M}^{\mathrm{r}}$ Sun[d]by me donne à penser et je crains que la leçon ne recommence.

St. Pétersbourg, Université. Le 7/19 mars 1877.

BNF, f. 122-123.

Alexandre Wesselofsky à Gaston Paris

28 juillet [18]77.

Cher Monsieur,

Il se pourrait que vous ou $\mathrm{M}^{\mathrm{r}}$ Meyer $^{21}$ trouvent de l'intérêt à une notice que j'ai prise, il y a une dizaine d'années, d'un fragment de Las Novas del papagay. Je ne crois pas qu'il vaille la peine d'être publié, mais puisque je l'ai retrouvé dans mes papiers, j'ai cru qu'il serait utile de le signaler ${ }^{22}$.

J'attends de Champion la suite des publications des Anciens textes ; en attendant je ne suis arrivé qu'à Brun de la Montagne23.

Je suis, Monsieur, votre très dévoué

Alexandre Wesselofsky.

BNF,f. 124

Gaston Paris à Alexandre Wesselofsky

Avenay ${ }^{24}$, ce 15 septembre 1882.

Cher Monsieur et ami,

Cette lettre vous sera remise par Monsieur Maurice Tourneux, que je prends la liberté de recommander à votre bienveillant accueil. II est chargé par notre Ministère d'une mission à Saint-Pétersbourg et à Moscou pour y examiner les livres et

21. Paul Meyer (1840-1917) collègue et ami de G. Paris, professeur, puis directeur de l'École des Chartes, professeur au Collège de France, membre de l'Académie des inscriptions et belleslettres (1884).

22. Ce texte fut publié par Veselovskij dans Romania (t. VII, n 26, 1878, p. 327-329).

23. Honoré Champion (1846-1913). Il s'agit du libraire parisien qui fut correspondant de Veselovskij (15, quai Malaquais). Il éditait des ouvrages d'histoire et de lettres, ainsi que la collection «Anciens textes français », dont Brun de la Montagne, roman d'aventures publié pour la première fois d'après le manuscrit de Paris par Paul Meyer(1875).

24. Avenay (Marne), ville natale de G. Paris. 
papiers de Diderot (dont il a donné la dernière et excellente édition), en dresser 1 'inventaire exact, et voir s'il s'y trouve encore quelque chose d'inédit et d'intéressant. Je vous serai très obligé de ce que vous pourrez faire pour lui faciliter sa tâche, et je vous demanderai particulièrement de 1'adresser à Moscou à ceux de vos amis qui puissent le guider le plus utilement ${ }^{25}$.

Je profite de cette occasion pour vous remercier de 1'attention que vous avez de m'envoyer un excellent article, que je ne verrais pas sans cela; vous en notez sans doute dans la Romania 1'accusé de reception ${ }^{26}$. Ne nous donnerez-vous pas quelque article un de ces jours ? Je serais heureux de savoir que mon premier article sur la Table Ronde vous paraît plausible ; je vais poursuivre ces recherches pendant plus d'une année 27 . J'ai bien des choses intéressantes à dire sur Merlin²8, et je profiterai alors de votre beau livre ; je 1'ai malheureusement prêté il y a longtemps à quelqu'un qui ne me 1'a pas rendu; mais je sais où je puis le retrouver ${ }^{29}$. Avez-vous quelque grand travail sur le chantier?

Croyez bien, cher Monsieur et ami, à mes sentiments tout dévoués.

\section{G. Paris.}

11 , rue de Varenne.

MP, 1. 1-2.

25. Maurice Tourneux (1849-1917), historien. En 1875-1877 il publia avec J. Assézat les Euvres complètes en vingt volumes de Denis Diderot. Les buts de son séjour à Saint-Pétersbourg et à Moscou étaient de « dresser un catalogue des manuscrits de Diderot, y rechercher les traces de sa bibliothèque et préparer ainsi les éléments d'une édition définitive de ses œuvres » (M. Tourneux, «Les manuscrits de Diderot conservés en Russie », Archives des missions scientifiques et littéraires, 3-e sér., t. XII., P., 1885, p. 439-474.

26. Il appert d'une lettre de P. Meyer à Veselovskij du 2 janvier 1882 (MP, f. 45, inv. 3, $\left.N^{\circ} 524,1.1-2\right)$ que Veselovskij avait adressé à G. Paris en 1881 un tiré à part de son article «Croissans-crescens i srednevekovye legendy o polovoj metamorfoze » (Croissans-crescens et les légendes médiévales sur la métamorphose sexuelle).

27. Il s'agit du premier article de Paris sur les romans du cycle arthurien : «Études sur les romans de la Table Ronde. Lancelot du Lac. I. Le Lanzelet d'Ulrich de Zatzikhoven » (Romania, t. X, 1881, p. 465-476).

28. À cette époque, G. Paris, de concert avec Jacob Ulrich, travaillait à l'édition d'un des romans (Merlin) du cycle du roi Arthur. Ce travail fut achevé en 1883, mais vit le jour beaucoup plus tard : Merlin, roman en prose du XIII-e siècle publié [...] par Gaston Paris et Jacob Ulrich, P., 1886, 2 vol.

29. Il s'agit de la thèse de doctorat de Veselovskij : Iz istorii literaturnogo obščenija Vostoka $i$ Zapada: Slavjanskie skazanija o Solomone $i$ Kitovrase $i$ zapadnye legendy o Morol'fe $i$ Merline [Un épisode de l'histoire des contacts littéraires entre 1'Orient et l'Occident : Les dits slaves sur Salomon et Kitovras et les légendes occidentales sur Morolf et Merlin], SPb., 1872. 
Alexandre Wesselofsky à Gaston Paris

22 janvier [18]83.

Cher Monsieur et ami,

Je n'ai pas eu de la chance avec Monsieur Tourneux. Il m'a fait remettre votre lettre par mon collègue à l'Académie $\mathrm{M}^{\mathrm{r}}$ Grote $^{30}$, et comme j'avais justement alors des malades à la maison, je n'ai pu faire ma visite à $\mathrm{M}^{\mathrm{r}}$ Tourneux qu'une dizaine de jours après. Je ne l'ai pas trouvé à la maison et ne l'ai pas revu depuis. Serait-il parti pour Moscou ? Il m'est extrêmement désagréable que les choses se soient passées ainsi et que je n'aie pas eu l'occasion d'être utile à $\mathrm{M}^{\mathrm{r}}$ Tourneux. Je sais d'ailleurs qu'il a été très bien recommandé et $\mathrm{p}<\mathrm{eut}>$-ê $<$ tre $>$ mieux que je ne l'aurais pu faire.

Je suis avec un grand intérêt vos études sur les romans de la Table Ronde. Malheureusement Champion, qui est mon libraire-correspondant à Paris, ne m'a pas encore envoyé les $\mathrm{N}^{\circ} \mathrm{N}^{\circ}$ de la Romania, qui ont paru depuis le $\mathrm{N}^{\circ} 40$. Il faut que je lui écrive. Et puisque je viens de toucher la question vexée de mes desiderata, permettez-moi de vous adresser une demande, que depuis plusieurs années j'adresse à Champion, quoique inutilement : il s'agit du $\mathrm{N}^{\circ} 33$ de la Romania qui probablement s'est perdu en chemin et ne m'a jamais été remis. Il est vrai que j'ai pu me servir de l'exemplaire d'un abonné de mes amis ; mais comme ma collection demeurait incomplète, j'ai prié Champion de me procurer le $\mathrm{N}^{\circ} 33$ « auf antiquarischem Wege », comme disent les Allemands, et contre remboursement. Il y a bien deux ans que j'attends la réponse ; y aurait-il quelques moyens d'y pourvoir?

Si vous avez besoin d'un exemplaire de mon livre sur Salomon et Kitovras etc., je pourrais vous l'offrir, pourvu que vous m'écriviez deux mots. Mais <c'est $>$ bien là une anticaille, que j'ai dépassée moi-même (en bien ou en mal) sur bien des points. Vous auriez pu le voir de mon compte-rendu du livre de Vogt, qui s'est approprié les résultats de mon travail, vieux de dix ans ${ }^{31}$. Le $1^{\mathrm{r}}$ article a paru dans l'Archiv f<ür> Slavische Philologie VI, le $2^{\mathrm{d}}$ va paraître prochainement. L'un et l'autre ont été traduits par Mr Wolter du texte russe de mes «Recherches sur les sources des cantiques pieux du peuple russe » (geistliche Lieder), livr<aison> III (1881). Je vous ai envoyé, il y a bien longtemps, les deux premiers fascicules (Légendes de st. Théodore et st. Georges) et je pourrais vous offrir le $3 \mathrm{e}$ et le $4 \mathrm{e}$, dont 23 feuilles sont déjà imprimées ${ }^{32}$. En en relisant les dernières épreuves je m’aperçois que j'y ai énoncé une foule d'hypothèses, les unes plus hasardées que les

30. Jakov Karlovič Grot (1812-1893), membre de l'Académie des sciences de Russie (1855).

31. Il s'agit de l'ouvrage de Fr. Vogt, Die deutsche Dichtungen von Salomon und Markolf. 1. Band : Salman und Morolf, Halle, 1880.

32. Razyskanija v oblasti russkih duhovnyh stihov [Recherches dans le domaine de la poésie religieuse russe] (1880-1881). La traduction en allemand des articles cités, publiée pour la revue Archiv für Slavische Philologie fut l'œuvre d'Eduard Aleksandrovič Vol'ter (18461941), linguiste, historien et ethnographe pétersbourgeois, plus tard professeur à l'université de Kaunas. 
autres. Je me consolerai, si la critique, en écartant ce qui y est de trop, y trouve matière à des recherches ultérieures.

En vous priant de saluer de ma part $\mathrm{M}^{\mathrm{r}}$ Paul Meyer, je vous prie, cher Monsieur et ami, d'agréer mes sentiments tout dévoués.

A.Wesselofsky.

BNF, f. 125-126.

Gaston Paris à Alexandre Wesselofsky

[6 février 1883.]

Cher Monsieur,

j'ai fait passer votre requête à Champion, en y insistant vivement ; s'il ne vous satisfait pas, je vous ferai envoyer le $\mathrm{n}^{\circ} 33$ par Vieweg ${ }^{33}$. J'accepte avec reconnaissance l'offre d'un exemplaire de «Sal<omon> et Kitovras », le mien s'étant perdu, je ne puis deviner par quel accident. J'ai lu votre 1-er article dans l'Archiv et il m'a vivement intéressé ; j'ai moi-même quelque chose à dire sur ce sujet. Vous verrez dans la Romania que j'ai parlé de vos premières études sur les cantiques russes ${ }^{34}$, et je suis heureux d'avoir la suite, quoique, hélas ! la lecture du russe me fût bien pénible. Je n'ai pas donné depuis le $\mathrm{n}^{\circ} 40$ d'article sur le roman breton ; je viens de finir le second (sur le roman de la Charette), qui paraîtra dans le nº d'août 83 (46) ${ }^{35}$. Je pense vous avoir envoyé le tirage à part du $1^{\mathrm{er}}$; sinon je vous enverrai les 2 ensemble. J'ai fait vos amitiés à Meyer, qui vous envoie les siennes ; sa santé m'inquiète un peu. Merci et bien à vous

G. Paris.

MP, 1.3

33. Friedrich Vieweg, libraire-éditeur parisien, le propriétaire-gérant de Romania.

34. Romania, t. X, n³7, 1881, p. 319-320.

35. «Études sur les romans de la Table Ronde. Lancelot du Lac. II Le conte de la Charette » (Romania, t. XII, n 46, 1883, p. 459-534). 
Gaston Paris à Alexandre Wesselofsy

\author{
11 , rue de Varenne.
}

Ce 25 avril 1884.

Cher Monsieur,

Pardonnez-moi d'avoir tant tardé à vous répondre, cher ami, <c'est ainsi,> hélas ! que commencent toutes mes lettres. J'ai reçu votre intéressant article, et je ne 1'ai pas remis à Chuquet $^{36}$, je l'ai, sauf votre agrément, bel et bien gardé pour la Romania où il convient mieux et par la dimension et par ce fait que vous y aviez déjà publié votre beau travail sur Constantin. Il paraitra en juillet, dans un $n^{\circ}$ double (avril-juillet) ${ }^{37}$.

J'ai toujours à vous faire les plus vifs remerciements pour les envois que vous me faites de vos articles ; je les parcours, et je vous promets, quand j'aurai du loisir, de les étudier à fond, un dictionnaire à la main. Je vous envoie, non en échange, une bluette qui aura peut-être quelque intérêt pour vous, et qui a au moins un mérite, c'est d'être une rareté, n'étant pas dans le commerce ${ }^{38}$.

L'Alexandre de Meyer est à peu près terminé, mais Dieu sait quand il paraîtra ! Il dit qu'on le verra cet été, j'en doute fort, et je vous engage à ne pas 1'attendre pour imprimer vos recherches ${ }^{39}$.

Je me recommande toujours à votre bon souvenir, et vous prie de penser à 1 'occasion à la Romania.

Bien à vous

G. Paris.

MP, 1. 4-5

36. Arthur Chuquet (1853-1925), historien, secrétaire et plus tard directeur de la Revue critique d'histoire et de littérature.

37. La recension par Veselovskij, et sur la proposition de G. Paris, pour la Revue critique d'histoire et de littérature, de l'ouvrage d'Achille Coen, Di una leggenda relativa alla nascita e alla gioventù di Costantino Magno (Roma : Jorzani, 1882), fut publiée dans Romania (t. XII, $\mathrm{n}^{\circ} 53,1885$, p. 137-143).

38. Il s'agit peut-être d'un tiré à part de l'article de G. Paris publié dans Romania (1882) : « Le Carmen de Prodicione guenonis et la légende de Roncevaux »; dans la Bibliographie de la France, il est mentionné comme une édition à part au 31 mars 1883 (n³269) avec la mention «non mis dans le commerce ».

39. Il s'agit du cycle «K voprosu ob istočnikah Serbskoj Aleksandrii » [À propos des sources de l'Alexandrie serbe], d'abord publié dans Žurnal ministerstva narodnogo prosveščenija en 1884-1885, puis dans le premier tome de l'ouvrage Iz istorii romana i povesti [Pages d'histoire du roman et du récit], 1886. La recherche de P. Meyer dont il est question fut commencée en 1866. Le premier volume fut publié en 1870 , la préparation du second se prolongea pendant plusieurs années et l'ouvrage complet parut en 1886 : Paul Meyer, Alexandre le Grand dans la littérature française du Moyen Âge, P., 1886, 2 vol. Veselovskij publia une recension de cette œuvre dans le Giornale storico della letteratura italiana (v. IX, fasc. 25-26, 1887, p. 255-266). Meyer lui répondit par une lettre de remerciements dans laquelle il exprimait l'espoir de prendre rapidement connaissance des travaux de son critique dans ce domaine (MP, f. 45, inv. $\left.3, n^{\circ} 524,1.3-4\right)$. 
Gaston Paris à Alexandre Wesselofsky

11 , rue de Varenne

[ Juillet 1884.]

Cher Monsieur,

Je vous ai envoyé, il y a déjà quelque temps, l'épreuve, avec la copie, de votre article sur Coen que j'ai, comme je vous en avais prévenu, gardé pour la Romania. - Je n'ai pas reçu votre épreuve corrigée, et n'ayant pas le manuscrit, il m'est difficile de le corriger moi-même. Je vous en envoie un exemplaire, en vous priant de me le retourner immédiatement avec vos corrections, sans quoi je me verrais obligé de le renvoyer au $\mathrm{N}^{\circ}$ d'octobre ${ }^{40}$.

Pardonnez-moi mon importunité et croyez-moi bien

Votre dévoué

G Paris.

Envoyez la correction à la Bourboule (Puy-de-Dôme). Si vous m'aviez déjà adressé l'épreuve corrigée, ne prenez pas la peine de réitérer, à moins que vous ne l'eûssiez envoyée antérieurement au 23 juin ou environ, car dans ce cas elle se serait perdue à la poste.

MP,1.11.

11

Gaston Paris à Alexandre Wesselofsky

[Juillet-août 1885.]

Villa Bormettes.

Le Pouliguen.

Loire-Inférieure.

Mon cher ami,

Je suis bien sensible à vos aimables félicitations, et à votre projet plus aimable encore de faire durer par une petite publication per nozze le souvenir de la part que vous voulez bien prendre à l'heureux changement de ma vie ${ }^{41}$. J'ai annoncé mon mariage si peu de temps avant le jour où il s'est célébré que plusieurs de mes amis qui auraient eu la même intention y ont renoncé faute de temps. J'ai cependant reçu quatre de ces charmants témoignages, de Meyer, Darmsteter, Chatelain et Pitrè. Je

40. Voir note 36.

41. Il s'agit du mariage entre Gaston Paris et Marie Talbot. 
vous envoie la plaquette de Meyer qui est un vrai petit chef-d'œuvre et qui a un intérêt littéraire que vous appréciere ${ }^{42}$.

Je crois bien que j'ai reçu votre second fascicule sur les bylines jougo-slaves, mais je n'en suis pas tout à fait sûr, ayant été hors de Paris depuis plus d'un mois, et depuis plus longtemps encore hors de mes livres et de mes travaux. Je m'assurerai du fait à mon retour, vous pensez que je tiens à ne pas perdre un présent de cette valeur ${ }^{43}$.

Je vous remercie encore bien cordialement, mon cher ami, et vous prie de me croire toujours très sincèrement

Votre tout dévoué

G. Paris.

à Paris, 110, rue du Bac.

MP, 1. 10.

Gaston Paris à Alexandre Wesselofsky

[ début novembre 1886.]

Cher Monsieur,

J'apprends par un mot de M. Cosquin ${ }^{44}$ que vous êtes à Meran ${ }^{45}$, où vous retient la santé de votre femme ${ }^{46}$, et je me hâte de vous y écrire. Je vous avais envoyé une lettre à Saint-Pétersbourg par un ami qui vient de partir pour la Russie et qui aurait été heureux de vous être présenté; il vous la rapportera, car il ne fait que passer quelques jours dans vos deux capitales. J'ai trouvé ici en revenant de la campagne le charmant souvenir que vous m'avez envoyé, et dont j'ai été, comme vous pouvez le croire, très fier et très touché ${ }^{47}$. Cette légende soulève, comme vous l'indiquez,

42. Voici l'annonce qui en fut faite dans Romania (t. XIV, n 56, 1885, p. 620-621) : « L'un des directeurs de la Romania ayant contracté mariage à Avenay (Marne), le 20 juillet dernier, cet heureux événement a donné lieu, suivant un usage italien bien connu de nos lecteurs et qui venait d'être inauguré en France [...], à quatre petites publications, tirées à fort peu d'exemplaires, et que nous croyons bon de signaler. Les voici: 1) Manuscrits de la bibliothèque de l'Université tirés des depôts littéraires [...]2) Note sur l'histoire des prépositions françaises en, enz, dedans, dans, par Arsène Darmsteter [...] 3) La pistola que fon tramesa an Gaston Paris le jorn que pres molher de part lo sien bon amic [...] [par P. Meyer] 4) Sonatori, balli e canti nuziali del popolo siciliano, per Giuseppe Pitrè [...].

43. Cycle : Južno-russkie byliny [Les byliny de la Russie du Sud] (vyp. III-XI, 1884).

44. Emmanuel Cosquin (1841-1919) folkloriste, auteur de Contes populaires de Lorraine, comparés avec les contes des autres provinces de France et des pays étrangers et précédés d'un essai sur l'origine et la propagation des contes populaires européens, P., 1886, 2 vol.

45. Meran, petite ville de cure dans le Tyrol, actuellement Merano en Italie.

46. L'épouse de Veselovskij, Elena Aleksandrovna, née Gen (1848-1906), avait une santé fragile.

47. Veselovskij a dédié « à Mr Gaston Paris en souvenir du 20 juillet 1885 » un fragment du premier tome de son cycle Iz istorii romana i povesti (op. cit.) intitulé : « O Tavre i Menii i ob osnovanii goroda », pour lequel on fit imprimer spécialement une page de titre (1886). 
des questions fort curieuses ; je crois depuis longtemps que 1'Italie du sud, aux IXe-XI-e siècles, a été le grand intermédiaire intellectuel entre le monde byzantin et le monde latin. Vous avez la même pensée et je m'en réjouis, car j'espère que vous la mettrez en commerce par des travaux que personne ne peut faire aussi bien que vous, et qui serait d'une très grande importance pour 1'histoire littéraire du moyen âge. Je ne puis vraiment assez vous remercier d'avoir si amicalement attaché mon nom à cette petite publication. Vous seriez bien aimable de me dire à qui, en dehors de moi, parmi nos amis communs d'Allemagne et d'Italie, vous l'avez envoyé. J'en ai donné des exemplaires à ceux d'ici que je croyais pouvoir y prendre intérêt, ainsi qu'à notre Académie ${ }^{48}$, il m'en reste de votre riche envoi, que j'aimerais adresser à des savants étrangers; mais je ne voudrais pas faire double emploi.

Cosquin m'écrit que vous ferez un article sur son livre ; j'en suis bien aise. C'est un ouvrage remarquable, et les vues générales, sauf quelques exagérations et quelques lacunes, sont justes et bonnes à répandre ${ }^{49}$.

J'espère que la santé de Madame Wesselofsky se trouve bien de votre séjour en Tyrol. Ne repasserez-vous pas par ici ? Ma femme serait heureuse de vous remercier aussi d'un présent dont elle a sa part. Mille cordiales amitiés.

G. Paris

MP, 1.13

\section{3}

Alexandre Wesselofsky à Gaston Paris

18 novembre [1886].

Cher Monsieur,

Il y a bien longtemps que l'opuscule que vous venez de recevoir a été imaginé et même mis à exécution, ma pensée étant de vous le faire parvenir à l'anniversaire de votre noce. Je comptai sans la maladie de ma femme et je partis à la campagne, laissant à un ami le soin de surveiller l'impression, qui a traîné jusqu'au mois de septembre. C'était le moment du départ, j'étais pressé et c'est comme cela que l'envoi vous est parvenu sans que j'aie eu le temps de vous l'annoncer ${ }^{50}$.

Je suis à Meran depuis à peu près un mois ; ma femme paraît s'y rétablir du mal de nerfs, dont elle souffre depuis quelque temps. Quant à moi, je me trouve ici comme dépaysé : en fait d'intérêt scientifique il n'y a que les ladins du voisinage,

48. L'Académie des inscriptions et belles-lettres.

49. Veselovskij écrivit à Cosquin entre le 1er et le 14 novembre 1886 et lui signifia son accord pour écrire une recension de son livre (MP, f. 45, inv. 3, $n^{\circ} 425,1.1-2$ ). Ce compte-rendu très développé fut publié dans Žurnal ministerstva narodnogo prosveščenija, vol. 250, avril 1887, p. 285-303.

50. Voir la note 46 . 
qui ne m'intéressent que médiocrément ${ }^{51}$, il faut que je me fasse venir chaque bagatelle de Leipzig ou de Vienne, car on ne trouve rien sur place. J'ai demandé à mon correspondant de Leipzig (Hæssel) ${ }^{52}$, le livre de $\mathrm{M}^{\mathrm{r}}$ Paul Meyer sur les romans d'Alexandre (annoncé dans la Romania), mais il y a bien deux semaines que je l'attends ${ }^{53}$.

Pour le moment j'ai en mains un petit travail sur un Tristan russe, encore inédit, le seul qui existe à ma connaissance, traduit du serbe (texte perdu ou non retrouvé), dont l'original a dû être italien et différait du texte de Polidori (Tavola Ritonda) en tant qu'il suit assez servilement le roman français en prose. La concordance avec ce dernier (et la Tavola Ritonda) ${ }^{54}$ cesse à l'épisode de Tristan délivrant Iseult des mains de Palamédès que celui-ci emmenait avec soi. Tout le reste est raconté différemment et sur un plan tout à fait divergent. J'ai demandé à Rajna s'il connaissait un texte pareil au mien ${ }^{55}$; mais il faudrait aussi voir le roman français (éd. de 1489 et 1520) dont je ne possède que des extraits. Puisqu'il n'y a pas moyen d'en avoir ici un exemplaire, j'en demanderai d'autres extraits à mon ancien élève, $\mathrm{Mr}^{\mathrm{r}}$ Braun $^{56}$, qui vient de faire ses études chez Paul, Brugmann et Neumann ${ }^{57}$ et se trouve pour le moment à Paris pour raisons de santé. Il aurait grand plaisir de faire votre connaissance, si toutefois vous permettez qu'il se présente chez vous ${ }^{58}$.

Mes saluts respectueux à Madame Paris, et n'oubliez pas de me rappeler à la mémoire de Monsieur Paul Meyer.

Je suis, Monsieur,

Votre très dévoué

Alexandre Wesselofsky.

Je ne me suis réservé que 7 exemplaires de la brochure que je vous ai transmise, et je l'ai distribuée parmi quelques amis de Pétersbourg.

BNF, f. 130-131.

51. Ladin, nom donné à un groupe de dialectes appartenant à la famille des langues romanes et parlés dans les régions rhétiques.

52. G. Hæssel : éditeur à Leipzig.

53. Voir la note 38.

54. La Tavola Ritonda o l'istoria di Tristano, testo di lingua publ. per cura di Filippo e Luigi Polidori. Bologna, 1864-1865, 3 vol.

55. Pio Rajna (1847-1930), spécialiste littéraire.

56. Fedor Aleksandrovič Braun (1862-1942), élève de Veselovskij, spécialiste de littératures germaniques, privat-dozent depuis 1888, puis professeur de 1900 à 1920 à l'université de SaintPétersbourg, enfin professeur à l'université de Leipzig à partir de 1923.

57. Hermann Paul (1846-1927), Karl Friedrich Brugmann (1849-1919), Fritz Neumann, philologues allemands.

58. G. Paris accepta cette prière de Veselovskij et, mieux encore, autorisa Braun à assister à ses cours au Collège de France et à l'École pratique des hautes études. 
Gaston Paris à Alexandre Wesselofsky

[25 ou 26 avril 1887]

Cher Monsieur,

J'ai un assez gros travail à vous envoyer; quoiqu'il ne fût guère approfondi et qu'il fût, par les circonstances, bien incomplet, il pourra avoir quelque intérêt pour vous $^{59}$. Mais je ne sais au juste où vous prendre, et je ne voudrais pas que mon volume se perdît. Veuillez me donner votre adresse actuelle ${ }^{60}$, et je vous 1 'expédierai. J'espère que la santé de Madame Wesselofsky s'est raffermie, et que votre voyage aura été utile.

Bien à vous

G. Paris.

Веселовский, профессор в Үниверситете

Monsieur A. Wesselovsky, professeur à l'Université,

Saint-Pétersbourg (Russie)

MP,f. 6.

15

[Carte de visite]

[mai 1890.]

\section{Gaston Paris \\ Membre de l'Institut}

présente à M. Paul Stapfer ${ }^{61}$, avec ses meilleurs compliments son ami le professeur A. Wesselofsky de Saint-Pétersbourg.

110 , rue du Bac.

MP, $\mathrm{N}^{\circ} 875,1.3$.

59. Gaston Paris, La poésie du Moyen Âge. Leçons et lectures, $1^{\text {e }}$ sér,. P., 1887.

60. Les Veselovskij se trouvaient alors à Meran.

61. Paul Stapfer (1840-1917), professeur d'histoire littéraire à l'université de Bordeaux. 


\section{6}

Alexandre Wesselofsky à Gaston Paris

14 juin 1890.

Cher Monsieur,

Je vous remercie de vos bons offices auprès de $\mathrm{M}^{\mathrm{r}} \mathrm{Picot}$, auquel je viens d'écrire, en lui transmettant la somme de 300 francs $^{62}$.

J'ai trouvé à Arcachon beaucoup de vent et de pluie - et pas de moyen pour me désennuyer en travaillant. Pour une petite notice que je voudrais faire pour la Romania, j'aurais besoin de quelques ouvrages et articles, que je vois mentionnés dans une note bibliographique de G. Paris, Litt<érature> franç<aise>, 2 éd., p. 284, § 150: Bibliotheca Normannica II (Wolter, Der Judenknabe) ; $Z<$ eit $>$ S $<$ chrift $>$ für roman<ische $>$ Philologie IX, 412 (si pourtant il y a des faits nouveaux concernant la légende même) et Mélusine V (sans autre indication chez G. Paris) ${ }^{63}$. Si je n'avais pas l'intention d'aller un de ces jours en Italie pour un séjour de 2-3 semaines, j'aurais pris au mot Mr Paris, qui m'a promis de me fournir de temps en temps des livres à mon usage. Je compte pouvoir profiter plus tard de son offre si aimable.

Agréez, cher Monsieur, l'assurance de mes sentiments les plus dévoués.

Alexandre Wesselofsky.

BNF, 1. 127. Le contenu de cette lettre et, en particulier la mention, à plusieurs reprises, de Gaston Paris à la troisième personne permettent de supposer que son destinataire n'était pas lui, mais un autre collègue.

Alexandre Wesselofsky à Gaston Paris

18 juillet [1890].

Cher Monsieur,

De retour de mon voyage d'Italie, qui m'a valu une grosse dyspepsie, je trouve une lettre de mon jeune ami, Monsieur Constantin Feuillye ${ }^{64}$, Français, né en

62. Émile Picot (1844-1918), historien médiéviste, membre de l'Académie des sciences morales et politiques (1897).

63. Gaston Paris, Manuel d'ancien français. La littérature française au Moyen Âge (XI XIVe siècles). Deuxième édition [...] ; P., 1890 ; Mélusine, revue de « mythologie, littérature populaire, traditions et usages », fondée en 1877 par Henri Gaidoz et Eugène Rolland.

64. Konstantin Ljucianovič Fel'i (Feuilly). Élève de Veselovskij, diplômé en 1886. Il passa l'été 1890 à Paris (voir MP, f. 45, inv. 3, N 769). 
Russie, élève de $\mathrm{M}^{\mathrm{r}}$ Batiouchkof ${ }^{65}$ (pendant mon absence de Pétersbourg il y a cinq ans) et le mien. Si vous vouliez bien lui faciliter l'accès des bibliothèques (et de la Section des Mss.), je vous en serais bien reconnaissant. $\mathrm{M}^{\mathrm{r}}$ Feuillye a déjà fait preuve de ses connaissances paléographiques en copiant pour $\mathrm{Mr}$ le prof. Constans quelques pages du Roman de Troie (Ms. de St. Pétersbourg) ${ }^{66}$.

La lettre de Mr Feuillye est datée du 3 juillet (Paris) et nous sommes le 18 ; il est donc possible que la lettre que je lui écris présentement, en lui indiquant votre adresse, ne le trouve plus à Paris. Dans ce cas-là je serais en retard, ce qui n'est pas ma faute.

Voudriez-vous bien me prêter Wolter, Der Judenknabe (Bibl<iotheca> Normannica, éd. Suchier II) ?67 Je songe toujours à une petite étude pour la Romania $^{68}$. Je me passerais pour le moment de quelques textes de la légende, indiqués dans la Zeitschrift IX (Alfonso X, cantigas $\mathrm{N}^{\circ}$ IV ; Libro de los enxemplos $\mathrm{N}^{\circ} \mathrm{CC}$; Horstmann dans Herrigs Arch. LVI, 228 ; Giorn<ale> stor<ico $>$ d <ella $>$ letter $<$ atura $>$ italiana III, $412 \mathrm{e}<\mathrm{t}>$ Ulrich, Romania, XIII, 54), mais je pourrais compléter l'étude lors de mon passage à Paris.

A peine rétabli, j'irai voir Monsieur André Delaroche-Vernet ${ }^{69}$, qui m'a fait l'honneur d'une visite tandis que j'étais absent. Je ne suis pas sûr de le retrouver, mais je n'ai pas de chance cette fois : c'est comme cela que je n'ai pu voir mon ami D’Ancona, qui est allé à Biella (Andorno) ${ }^{70}$, n'ayant pas reçu les lettres, lui annonçant ma visite.

Je suis, cher Monsieur, votre très dévoué

Alexandre Wesselofsky.

BNF, f. 128-129

65. Fedor Dmitrievič Batjuškov (1857-1920) élève et collaborateur de Veselovskij, historien de la littérature, critique, homme de théâtre.

66. Léopold-Eugène Constans (1845-1916), professeur de langue et de littérature provençales à l'université d'Aix-en-Provence. Il s'agit de son grand ouvrage, Le Roman de Troie, par Benoît de Sainte-Maure, publié d'après tous les manuscrits connus [...], P., 1904-1912, 6 vol.

67. Der Judenknabe, hrsg von Eugenius Wolter, Halle, 1879 (Bibliotheca Normannica. Denkmäler normannischer Literatur und Sprache, hrsg von Hermann Suchier, II).

68. Après 1890 Veselovskij ne publia plus dans Romania.

69. André Delaroche-Vernet, membre de la famille de Marie Paris Talbot (veuve de Philippe Delaroche-Vernet).

70. Alessandro D’Ancona (1835-1914), spécialiste littéraire et homme politique italien, rédacteur de 1893 à la fin de sa vie de la revue Rassegna bibliografica della letteratura italiana. Biella, Andorno, stations balnéaires dans le Piémont (province de Vercelli). 
Gaston Paris à Alexandre Wesselofsky

Paris, 24 juillet [1890].

Mon bon ami,

Par un accident fâcheux, je lis votre lettre seulement aujourd'hui, en sorte que je ne l'avais pas lue, quand j'ai reçu hier M. Feuillye. Mais meno male ! je n'avais à faire pour lui que lui donner des indications très simples. Je vais monter en chemin de fer et serai demain à Saint-Nectaire (Puy-de-Dôme). Je passerai 3 jours à Arcachon vers le 15 août ; y serez-vous?

André est revenu depuis longtemps et il a bien regretté de ne pas vous trouver ${ }^{71}$. J'espère que vous allez tout à fait bien .

Je vous envoie le livre de Wolter ${ }^{72}$, et serai très heureux d'avoir votre article pour la Romania. Excusez la hâte et croyez-moi

Bien à vous

G. Paris.

MP,1.7.

[Carte de visite]

\section{Gaston Paris, de l'Académie française et de l'Académie des inscriptions et belles-lettres}

présente à M.A. Wesselofsky, avec ses meilleures amitiés Mademoiselle Obert, qui s'occupe de traduire du russe des contes de Renard et qui serait heureuse d'avoir de lui quelques renseignements ${ }^{73}$. Paris, 11 janvier 1899.

G. Paris.

Collège de France.

MP, $n^{\circ} 875,1.4$.

71. Voir la note 70 .

72. Voir la note 68.

73. M. Obert se servit de cette recommandation, car le 4 février 1899 , elle adressa cette lettre à Veselovskij : «Recommandée à votre bienveillance par Monsieur Gaston Paris, chargée par Monsieur Tobler de vous présenter ses compliments, j'ai le courage de vous prier de m'accorder le privilège d'une audience. Je vous serais profondément reconnaissante, Monsieur, si vous vouliez bien accéder à ma demande et me sacrifier quelques moments de votre temps précieux. Dans ce cas oserais-je vous prier de vouloir bien m'avertir du jour et de $l^{\prime}$ heure où je pourrais me rendre à vos ordres ? » (MP, f. 45, inv. 3, $\left.\mathrm{N}^{\circ} 574\right)$. Adolf Tobler, philologue allemand. 
Gaston Paris à Alexandre Wesselofsky

$$
\text { Cerisy-la-Salle, } 6 \text { août }[1899]^{74} \text {. }
$$

Mon cher confrère et ami,

Je suis bien profondément touché de voir votre nom sur la liste des amis qui viennent de m'offrir un présent si magnifique. C'est un honneur fort au-dessus de mes mérites, mais que $\mathrm{j}$ 'accepte avec une profonde reconnaissance à cause du sentiment qui en a inspiré les auteurs, et le fait que vous avez voulu y prendre part me le rend encore plus précieux ${ }^{75}$. Conservez-moi votre amitié et croyez-moi bien cordialement

Votre très dévoué

G. Paris

MP,1.9.

74. Le château de Cerisy-la Salle appartenait à la famille de la seconde épouse de Gaston Paris Marguerite Savary (ils s'étaient mariés le 10 septembre 1891).

75. La datation de cette lettre est hypothétique. Le 9 août 1899 Gaston Paris eut son soixantième anniversaire et Veselovskij ne pouvait laisser passer une telle date sans se manifester. 